\title{
COMPARAÇÃO DE MÉTODOS PARA DETERMINAÇÃO DE BIOMASSA MICROBIANA EM DOIS SOLOS(1)
}

\author{
M. M. ANDRÉA ${ }^{(2)} \&$ M. J . MORENO HOLLWEG ${ }^{(3)}$
}

\begin{abstract}
RESUMO
O potencial de medidas de biomassa microbiana do solo como indicadoras da bioatividade e das interferências nesse ambiente pode ser verificado pela quantidade de trabal hos publicados a esse respeito. Entretanto, a comparação entre dados de diferentes autores, localidades e solos só é possível quando o método de determinação é o mesmo. No entanto, há numerosos trabalhos que propõem diferentes métodos e cálculos. Este trabalho avaliou resultados de determinação de $\mathbf{C}$ microbiano obtidos por diferentes métodos de extração e digestão de amostras de dois solos e cálculos de biomassa; além de diferentes cálculos para transformação indireta de $\mathrm{CO}_{2}$ produzido por respiração basal e induzida em C microbiano, nos dois diferentes solos. Os resultados variaram bastante entre métodos e cálculos e concluiu-se que o método de Vance et al. (1987), mais freqüentemente citado na literatura, deve ser a opção para boa comparação da quantidade de $\mathbf{C}$ microbiano entre solos e dados da literatura.
\end{abstract}

Termos de indexação: carbono microbiano, extração, digestão, respiração.

\section{SUMMARY: COMPARISON OF METHODS FOR DETERMINING MICROBIAL BIOMASS IN TWO SOILS}

The potential of soil mi crobial biomass measurements as indicators of soil bioactivity and interferences in this environment can beverified by the amount of publ ished papers on the subject. Neverthel ess, a comparison among data from different authors, regions, and soils is only possible when the methodology employed in the determinations is the same Several papers, however, propose different methodologies and calculations. This study evaluated theresults of microbial carbon determi nation by different extraction and digestion

\footnotetext{
(1) Trabal ho parcialmente apresentado no XXIX Congresso Brasileiro de Ciência do Solo (julho/2003). Recebido para publicação em agosto de 2003 e aprovado em outubro de 2004.

(2) Pesquisador Científico, Instituto Biológico, Centro de Proteção Ambiental, Lab. de Ecologia de Agroquímicos, Caixa Postal 12898, CEP 04010-970 São Paulo (SP). E.mail: andrea@biologico.sp.gov.br

(3) Graduanda do Instituto de Biociências, USP. Rua do Matão, Travessa 14, no 321, CEP 05508-900 São Paulo (SP) E-mail: mariaju@hotmail.com
} 
methodol ogies in two distinct soils. It was also performed biomass calculations, besides calculations for indirect transformation of $\mathrm{CO}_{2}$ from basal and induced respiration into microbial carbon in thetwo soi ls. Results varied greatly among methods and calculations, and the conclusion was drawn that the most frequently used methodology (Vance et al., 1987) should beadopted for a valid comparison of the amount of microbial carbon among soils and literature data.

Index terms: microbial carbon, extraction, digestion, respiration.

\section{NTRODUÇÃO}

Práticas agriculturais, tais como: tipo de manejo do solo, aração, rotação de culturas e aplicação de agrotóxicos, interferem na microbiota do solo. É amplamente conhecida a importância dos organismos edáficos na ciclagem de nutrientes necessários para crescimento de plantas e animais, além da sua atuação na manutenção das condiç̧̃̃es físico-químicas efertilidade desteambiente (Pagga, 1997). Sabe-se também que a microbiota é o principal agente na biodegradaçãotanto da biomassa disponível, quanto de xenobióticos que atingem ou são aplicados no sol o (Suett et al ., 1996; Pagga, 1997).

As variações nos números de indivíduos ou na dinâmica bioquímica natural da comunidade de microrganismos do solo, que podem ocorrer em conseqüência da ação antropogêni ca ou das variações sazonais naturais, podem ser medidas pela variação da biomassa microbiana epor variações de diferentes processos enzimáticos que podem servir como bioindicadores (Nielsen \& Winding, 2002).

Para avaliar o efeito de interferências nesse ambiente, há necessidade de utilização de métodos sensíveis que detectem pequenas mudanças. O isolamento de linhagens de organismos envolvidos nos processos de interesse de cada solo pode não representar situação generalizável porque, de modo geral, consegue-se isolamento de somente 1 a $3 \%$ dos microrganismos do solo (Lin \& Brookes, 1999), eas populações variam natural mente de acordo com as características pedogêni cas e variações climáticas locais (Berry, 1994; Suett et al., 1996). Por esse motivo, pesquisas sobre bi oindicadores têm utilizado medidas de propriedades mais genéricas, tais como medidas da respiração mi crobiana, mineralização de algumas substâncias e atividades de diferentes enzimas, que traduzem as reações não só de populações individuais, mas também de comunidades econsórcios (Moorman, 1994), além de apresentarem melhor reprodutividade (Schinner et al., 1996).

Além disso, a determinação da biomassa microbiana, por determinação do peso do C microbiano por unidade de peso do solo também tem sido utilizada como bioindicador e propriedade ecológica (Nielsen \& Winding, 2002). O primeiro trabalho a esse respeito foi feito por J enkinson \& Powlson (1976) e descreve o método de extração de C após fumigação de amostras de solo com vapor de clorofórmio e comparação com a quantidade de C extraído de amostras não fumigadas. O cálculo de transformação do C do solo para C microbiano foi descrito e adaptado por Vance et al. (1987). Entretanto, muitos pesquisadores propuseram mudanças no processo de fumigação e nos de extração e digestão dos extratos, assim como nos cál culos de $\mathrm{C}$ microbiano.

A biomassa microbiana do sol o também pode ser medida indiretamente por meio da respiração do solo (Nielsen \& Winding, 2002). M Medidas de respiração microbiana refletem diretamente a atividade de microrganismos heterótrofos e informam quanto à bi oatividade do sol o (Paul \& Clark, 1996). O método de determinação de respiração basal e induzida (SIR), originalmente proposto por Anderson \& Domsch (1978), permite converter dados de respiração em $C$ de biomassa, por aplicação de um fator de conversão aos resultados obtidos na respiração. O método baseia-se em medidas da respiração basal, que é a respiração real do solo, e da induzida, que é a respiração potencial, a partir da adição de um substrato de fácil metabolização, como a gl icose, geralmenteadicionada na proporção de $4 \mathrm{mg} \mathrm{g}^{-1}$ de glicose no solo (Lin \& Brookes, 1999). Vale ressaltar que os cál culos de transformação dos dados de respiração em $\mathrm{C}$ mi crobiano também foram modificados por outros autores.

Com o intuito de obter medida de biomassa microbiana que possa permitir comparação entre diferentes solos e com resultados de diferentes localidades e de diferentes autores, este trabalho comparou vários métodos propostos, em dois tipos de solos.

\section{MATERIAL E MÉTODOS}

\section{Processo de fumigação e extração de J enkinson \& Powlson (1976)}

A extração do C microbiano (C-mic) foi feita após fumigação $(F)$ de amostras de solo previamente umedecidas a $50 \%$ da Capacidade Máxima de RetençãodeÁgua, com vapor de $\mathrm{CHCl}_{3}$ livre deetanol, durante $24 \mathrm{~h}$. A retirada de etanol do $\mathrm{CHCl}_{3}$ foi feita, conforme Frighetto (2000). Após 24 h, o vapor do fumigante foi retirado e o $\mathrm{C}$ do solo (C-solo) foi extraído com $\mathrm{K}_{2} \mathrm{SO}_{4}$ 0,5 mol L-1. Como controle, 
foram usadas amostras de solo não fumigadas (NF). A seguir, os extratos $\mathrm{F}$ eNF foram digeridos a $100{ }^{\circ} \mathrm{C}$, após adição de $\mathrm{K}_{2} \mathrm{Cr}_{2} \mathrm{O}_{7} 0,0667 \mathrm{~mol} \mathrm{~L}^{-1}, \mathrm{HgO}$ e mistura de $\mathrm{H}_{2} \mathrm{SO}_{4} \mathrm{e} \mathrm{H}_{3} \mathrm{PO}_{4}(2: 1 \mathrm{v} / \mathrm{v})$.

O C-solo foi determinado por titulação dos extratos digeridos, após adição deindicador ferroína, com $\left[\left(\mathrm{NH}_{4}\right)_{2} \mathrm{Fe}\left(\mathrm{SO}_{4}\right)_{2} \times 6 \mathrm{H}_{2} \mathrm{O}\right] 0,0333 \mathrm{~mol} \mathrm{~L}^{-1}$ diluído em $\mathrm{H}_{2} \mathrm{SO}_{4} 0,4 \mathrm{~mol} \mathrm{~L}^{-1}$ e padronizado previamente por adição de ferroína e titulação com $\mathrm{K}_{2} \mathrm{Cr}_{2} \mathrm{O}_{7}$ 0,0667 mol L-1, água destilada $\mathrm{eH}_{2} \mathrm{SO}_{4}$ concentrado.

\section{Mudanças propostas por diferentes autores nos processos de extração e digestão:}

- Islam \& Weil (1998) empregaram irradiação com microondas em substituição à fumigação com $\mathrm{CHCl}_{3}$, e também para digestão dos extratos em substituição ao aquecimento. A determinação do C-mic foi feita por espectrometria na região da luz visível, em 590 nm, e comparada com curva-padrão de sacarose, para comparação com quantidades conhecidas de $\mathrm{C}$.

- Pascual et al. (2000): substituírama mistura $\mathrm{H}_{2} \mathrm{SO}_{4}$ e $\mathrm{H}_{3} \mathrm{PO}_{4}(2: 1 \mathrm{v} / \mathrm{v})$ utilizada para digestão dos extratos, por apenas $\mathrm{H}_{2} \mathrm{SO}_{4}$.

- Frighetto (2000) não adicionou HgO para a digestão dos extratos.

- Oliveira et al . (2001) utilizaram $\mathrm{K}_{2} \mathrm{Cr}_{2} \mathrm{O}_{7}$ 0,4 mol L-1 em vez de $0,0667 \mathrm{~mol} \mathrm{~L}^{-1}$ proposto por J enkinson \& Powlson (1976); proporção 1:2 (v/v) da mistura de ácidos $\mathrm{H}_{2} \mathrm{SO}_{4}: \mathrm{H}_{3} \mathrm{PO}_{4}$ e preparação da solução de $\left[\left(\mathrm{NH}_{4}\right)_{2} \mathrm{Fe}\left(\mathrm{SO}_{4}\right)_{2} \times 6 \mathrm{H}_{2} \mathrm{O}\right]$ por diluição em $\mathrm{H}_{2} \mathrm{SO}_{4}$ concentrado.

- D'Andréa et al. (2002) utilizaram proporção 1:2 (v/ v) da mistura de ácidos $\mathrm{H}_{2} \mathrm{SO}_{4}: \mathrm{H}_{3} \mathrm{PO}_{4}$.

Nenhum dos autores citados explica a razão de suas propostas de mudanças.

\section{Cálculos de C-mic do solo, conforme Vance et al. (1987):}

O C-micfoi calculado pela diferença do C-extraído do solo fumigado (C-solo F ) com o do solo não fumigado (C-solo NF), como C-extraível do solo em $\mu \mathrm{g}$ de $\mathrm{C} \mathrm{mL}^{-1}$ de extrato. Os cál culos realizados levaram em conta: os volumes de consumo de sol ução detitulação das amostras de sol ofumigado e controle não fumigado; os vol umes de consumo de solução de titulação dos controles de reagentes também submetidos aos processos de digestão e dos controles dereagentes não digeridos; a molaridade e o vol ume de $\mathrm{K}_{2} \mathrm{Cr}_{2} \mathrm{O}_{7}$ adicionado no processo de digestão; 0 volume de extrato de solo; o fator de conversão de redução $\mathrm{Cr}^{6+}$ para $\mathrm{Cr}^{3+}$ que ocorre durantea oxidação da matéria orgânica, para quantificação estequiométrica do dicromato remanescente nas titulações, e, finalmente, o fator de correção da padronização do $\left[\left(\mathrm{NH}_{4}\right)_{2} \mathrm{Fe}\left(\mathrm{SO}_{4}\right)_{2} \times 6 \quad \mathrm{H}_{2} \mathrm{O}\right]$ 0,0333 mol L-1, para expressar sua molaridadereal.
Os valores obtidos foram transformados em Csolo (em $\mu \mathrm{g}$ de $C \mathrm{~g}^{-1}$ de solo), levando em conta o volume do extrator $\left(\mathrm{K}_{2} \mathrm{SO}_{4} 0,5 \mathrm{~mol} \mathrm{~L}^{-1}\right)$, o peso das amostras e a percentagem de umidade do solo. Vance et al. (1987) chamaram de Ec essa diferença deC-solo F menos o C-solo NF e calcularam o C-mic para vários sol os com pH na faixa de 3,3 a 7,2, tendo encontrado uma relação linear entre o C-mic e Ec que permitiu a elaboração de equação de regressão, detal forma que os val ores de C-micforam cal culados segundo a seguinte fórmula:

$$
\begin{gathered}
\text { C-mic }\left(\mu g \mathrm{C} \mathrm{g}^{-1} \text { solo }\right)=2,64 \times \mathrm{Ec} \text {, ou: } \\
2,64 \times(\mathrm{C} \text {-solo F }- \text { C-solo NF })
\end{gathered}
$$

\section{Mudanças propostas por diferentes autores nos cálculos de $\mathbf{C}$-mic:}

- De acordo com Harden et al. (1993): C-mic = (Csolo F-C-solo NF) $\times 2,22$.

- De acordo com I slam \& Weil (1998): C-mic = (C-solo irradiado- C-solonãoirradiado) / 0,213. Os autores explicaram que 0,213 foi a fração de C da biomassa microbiana extraída por $\mathrm{K}_{2} \mathrm{SO}_{4} 0,5 \mathrm{~mol} \mathrm{~L}^{-1}$.

- De acordo com Hu et al. (1999) e Ferreira et al. (1999): C-mic $=(C-$-solo F-C-solo NF ) / 0,33.

- De acordo com Frighetto (2000): C-mic $=$ (C-solo F-C-solo NF ) / 0,38.

- De acordo com Croft et al. (2001): C-mic $=$ (C-solo F-C-solo NF ) / 0,45.

- De acordo com Ghani \& Wardle (2001): C-mic = (C-solo F-C-solo NF) / 0,40.

- De acordo com D'Andréa et al. (2002): C-mic =(Csolo F-C-solo NF ) / 0,30.

A mudança dos valores nos denominadores das fórmulas de cál culo não foi explicada nos trabalhos citados.

O método original deJ enkinson \& Powlson (1976) e os cálculos de Vance et al. (1987), baseados no método dos primeiros, bem como todas as modificações descritas, foram testados em pelo menos trếs repetições de cada um dos dois tipos de sol os estudados, cujos pesos das amostras variaram conforme o método.

\section{Método de respiração basal e induzida (SIR)}

O método empregado para verificação da respiração do solo baseou-se na produção de $\mathrm{CO}_{2}$ coletado periodicamente em $\mathrm{KOH} \mathrm{0,01} \mathrm{mol} \mathrm{L-1,}$ conformedescrito em Andréa \& Pettinelli J r. (2000). Anderson \& Domsch (1978) acharam correlação significativa entre as taxas de $\mathrm{CO}_{2}$ produzido e as de biomassas de vários solos, a qual permitiu calcular o C-mic dos sol os por mei o da transformação da taxa máxima de respiração inicial após adição de gl icose, em C-mic, utilizando a seguinte fórmula:

$$
\text { C-mic (mg de C-mic g }{ }^{1} \text { de solo) }=40,04 \text { y }+0,37
$$


em que y = taxa máxima de respiração inicial $(\mathrm{mL}$ de $\mathrm{CO}_{2} \mathrm{~g}^{-1}$ de solo $\mathrm{h}^{-1}$ ).

\section{Mudanças propostas nos cálculos de C-mic a partir de respostas respiratórias:}

- Segundo Sparling et al. (1990): C-mic ( $\mu$ g de C g-1 de solo $)=\left(\mu \mathrm{L}\right.$ de $\mathrm{CO}_{2} \mathrm{~g}^{-1}$ de solo $\left.\mathrm{h}^{-1}\right) \times 50$.

- Segundo Harden et al. (1993): C-mic ( $\mu \mathrm{g}$ de C $\mathrm{g}^{-1}$ de solo $)=\left(\mu \mathrm{L}\right.$ de $\mathrm{CO}_{2} \mathrm{~g}^{-1}$ de solo $\left.\mathrm{h}^{-1}\right) \times 30$.

A conversão dos dados col etados de $\mathrm{mg} \mathrm{CO}_{2} \mathrm{em}$ $\mu \mathrm{L} \mathrm{CO}$ foi feita por cálculos, sabendo-se que: $1 \mathrm{~mol}$ $\operatorname{de~}_{2}=22,4 \mathrm{~L}$ de $\mathrm{CO}_{2}$ e $1 \mathrm{~mol}$ de $\mathrm{CO}_{2}=44 \mathrm{~g} \mathrm{de} \mathrm{CO}_{2}$ (em CNTP).

As amostras dos solos submetidos aos testes foram coletadas de 0 a $15 \mathrm{~cm}$ do perfil dos solos de áreas agrícolas das regiões de Campinas e Barra Bonita, ambas no estado deSão Paulo. As principais características do solo argilo-arenoso de Campinas foram: $65 \%$ de areia, $15 \%$ de silte, $20 \%$ de argila, $31 \mathrm{~g} \mathrm{dm}^{-3}$ de matéria orgânica e pH $\left(\mathrm{CaCl}_{2}\right) 5,4$, de acordo com análise feita pelo Laboratório Lagro (Campinas, SP). As características do solo muito argiloso de Barra Bonita foram: $10,3 \%$ de areia, $28,1 \%$ desilte, $61,6 \%$ deargila, $25 \mathrm{~g} \mathrm{dm}^{-3}$ de matéria orgânica e $\mathrm{pH}\left(\mathrm{CaCl}_{2}\right)$ 5,0, de acordo com análise feita pelo Laboratório de Fertilidade do Solo da Faculdade de Ciências Agronômicas da UNESP/ Botucatu (SP).
Os resultados foram submetidos à análise de variância do teste $t(p<0,05)$ entre os diferentes métodos de digestão.

\section{RESULTADOS E DISCUSSÃO}

A quantificação de C-solo e, portanto, a de C-mic não foram possíveis pelo método de Oliveira et al. (2001) porque não se conseguiu titular os extratos com até $100 \mathrm{~mL}$ do sulfato ferroso amoniacal, razão por que não foi usada a fórmula de cál culo proposta por estes autores. Em relação aos demais (Quadro 1), observou-seque, independentemente do tipo de solo, os valores obtidos para um mesmo processo de digestão foram próximos e, com exceção dos cálculos propostos por Islam \& Weil (1998), independentes do tipo de cálculo. Por outro lado, diferentes processos de digestão determinaram grandes variações, isto é, de aproximadamente 7 a $90 \mu \mathrm{g} \mathrm{g}^{-1}$ de C-mic de solo de Campinas e de 6 a $54 \mu^{g^{-1}}$ de C-mic de solo de Barra Bonita (Quadro 1). Verificou-se que, assumindo ser o método padrão mais consagrado na literatura (Vance et al., 1987), apenas os cálcul os efetuados conforme Frighetto (2000) se aproximaram dele, enquanto os outros métodos de cál culos diferiram bastante. As maiores diferenças foram observadas com o método delslam \& Weil (1988), que utilizou microondas em substitui çãoà fumigação e na digestão das amostras.

\section{Quadro 1. Carbono da biomassa microbiana (C-mic) determinado por diferentes processos de digestão e extração de amostras do solo de Campinas e de Barra Bonita}

\begin{tabular}{|c|c|c|c|c|c|c|c|c|}
\hline \multirow[b]{2}{*}{ Digestão } & \multicolumn{8}{|c|}{ Cálculo } \\
\hline & $\begin{array}{c}\text { Vance } \\
\text { et al. } \\
(1987)\end{array}$ & $\begin{array}{c}\text { Harden } \\
\text { et al. } \\
\text { (1993) }\end{array}$ & $\begin{array}{c}\text { Islam \& } \\
\text { Wei I } \\
\text { (1998) }\end{array}$ & $\begin{array}{c}\text { Hu et al. } \\
\text { (1999) } \\
\text { Ferreira } \\
\text { et al. } \\
(1999)\end{array}$ & $\begin{array}{l}\text { Frighetto } \\
\text { (2000) }\end{array}$ & $\begin{array}{c}\text { Croft } \\
\text { et al. } \\
(2001)\end{array}$ & $\begin{array}{c}\text { Ghani \& } \\
\text { Wardle } \\
\text { (2001) }\end{array}$ & $\begin{array}{c}\text { D'Andréa } \\
\text { et al. } \\
\text { (2002) }\end{array}$ \\
\hline & \multicolumn{8}{|c|}{ - $\mathrm{mg} \mathrm{g}^{-1} \mathrm{C}-\mathrm{mic}$ de solo } \\
\hline & \multicolumn{8}{|c|}{ Campinas } \\
\hline Vance et al. (1987) & 71,47 & 60,10 & 20,66 & 82,04 & 71,24 & 60,16 & 67,68 & 90,24 \\
\hline Islam \& Weil (1998) & 11,62 & 9,77 & 20,66 & 13,33 & 11,58 & 9,78 & 11,00 & 14,67 \\
\hline Frighetto (2000) & 22,11 & 18,59 & 39,31 & 25,37 & 22,04 & 18,61 & 20,93 & 27,91 \\
\hline Pascual et al. (2000) & 15,53 & 13,06 & 27,62 & 17,83 & 15,48 & 13,07 & 14,71 & 19,61 \\
\hline Ghani \& Wardle (2001) & 38,23 & 32,15 & 67,99 & 43,88 & 38,11 & 32,18 & 36,20 & 48,27 \\
\hline \multirow[t]{2}{*}{ D'Andréa et al. (2002) } & 8,19 & 6,88 & 14,56 & 9,40 & 8,16 & 6,89 & 7,75 & 10,34 \\
\hline & \multicolumn{8}{|c|}{ Barra Bonita } \\
\hline Vance et al. (1987) & 29,41 & 24,73 & 52,30 & 33,76 & 29,32 & 24,76 & 27,85 & 37,13 \\
\hline Islam \& Weil (1998) & 2,59 & 2,18 & 4,60 & 2,97 & 2,58 & 2,18 & 2,45 & 3,27 \\
\hline Frighetto (2000) & 10,91 & 9,17 & 19,39 & 12,52 & 10,87 & 9,18 & 10,33 & 13,77 \\
\hline Pascual et al. (2000) & 7,12 & 5,99 & 12,67 & 8,18 & 7,10 & 6,00 & 6,75 & 9,00 \\
\hline Ghani \& Wardle (2001) & 30,24 & 25,43 & 53,79 & 34,72 & 30,15 & 25,46 & 28,64 & 38,19 \\
\hline D'Andréa et al. (2002) & 24,00 & 20,58 & 43,52 & 28,09 & 24,39 & 20,60 & 23,17 & 30,90 \\
\hline
\end{tabular}


Os resultados dos métodos de digestão diferiram significativamente dos de Vance et al. (1987) para todos os métodos utilizados com o sol o de Campinas, com valores de $p$ variando de 0,0001 (D'Andréa et al., 2002) a, no máximo, 0,0157 (Ghani \& Wardle, 2001). Com o sol o de Barra Bonita, só os resultados dos métodos de D'Andréa et al. (2002) e de Ghani \& Wardle(2001) não diferiram significativamente dos de Vance et al. (1987), com $p=0,1337$ e 0,7822, respectivamente.

Verificou-sequetodos os métodos subestimaram a quantidade de biomassa microbiana, quando comparados com os resultados obtidos por meio do método de Vance et al. (1987), tanto em relação à digestão, quanto em rel ação aos cálculos (Quadro 1). Notou-se que, embora a única diferença entre os métodos de digestão de Frighetto (2000) e de Vance et al. (1987) tenha sido a adição de $\mathrm{HgO}$ pelos últimos, este reagente determinou diferenças bastante grandes na biomassa das amostras dos sol os estudados. Enquanto o método deVance et al . (1987) determinou aproximadamente 71,5 e 29,4 . g g $^{-1}$ de C-mic de solo, respectivamente, nos sol os de Campinas e de Barra Bonita, o de Frighetto (2000) determinou, respectiva-mente, 22,1 e 10,9 .g g $^{-1}$ de C-mic de solo.

Quanto aos resultados de biomassa microbiana obtidos a partir de método de conversão de $\mathrm{CO}_{2}$ produzido por respiração basal e induzida, verificaram-se grandes diferenças nas quantidades obtidas, tanto em relação aos resultados de todos os demais métodos de digestão quanto em relação ao tipo de cál culo (Quadro 2). Este método, apesar de ser sensível e bastante usado como bioindicador em estudos dedinâmica de agrotóxicos em sol os (Harden et al., 1993; Andréa et al., 2000; Andréa \& Pettinelli J r., 2000; Andréa et al., 2003), não deve ser usado para cálculo de biomassa, já que os resultados também diferiram em relação aos obtidos pelo método de Vance et al. (1987).

\section{Quadro 2. Carbono da biomassa microbiana (C-mic) determinado por diferentes cálculos a partir de medidas de respiração basal e induzida em amostras do solo de Campinas e de Barra Bonita (mg C-mic g ${ }^{-1}$ solo)}

\begin{tabular}{lccc}
\hline Solo & $\begin{array}{c}\text { Anderson } \\
\text { \& Domsch } \\
\text { (1978) }\end{array}$ & $\begin{array}{c}\text { Sparling } \\
\text { et al. (1990) }\end{array}$ & $\begin{array}{c}\text { Harden } \\
\text { et al. (1993) }\end{array}$ \\
\hline Campinas & 730 & 445 & 267 \\
Barra Bonita & 749 & 469 & 281 \\
\hline
\end{tabular}

\section{CONCLUSÃO}

O método de Vance et al. é o método de escol ha para boa comparação entre os solos e os dados da literatura.

\section{LITERATURA CITADA}

ANDERSON, J.P.E. \& DOMSCH, K.H. A physiological method for the quantitative measurement of microbial biomass in soils. Soil Biol. Biochem., 10:215-221, 1978.

ANDRÉA, M.M.; PERES, T.B.\& MATALLO, M.B. Effect of the herbicide haloxyfop-methyl on some soil biological parameters. In: INTERNATIONAL WEED SCIENCE CONGRESS, 3., Foz do I guaçu, 2000. Proceedings. F oz do I guaçu, International Weed Science, 2000. CD-ROM

ANDRÉA, M.M. \& PETTINELLI J r., A. Efeito de aplicações de pesticidas sobre a biomassa e a respiração de microrganismos de sol os. Arq. Inst. Biol., 67:223-228, 2000.

ANDRÉA, M.M.; PERES, T.B.; LUCHINI, L.C.; BAZARIN, S.; PAPINI, S.; MATALLO, M.B. \& SAVOY, V.L.T. Influence of repeated applications of glyphosate on its persistence and soil bioactivity. Pesq. Agropec. Bras., 38:1329-1335, 2003.

BERRY, E.C. Earthworms and other fauna in the soil. In: HATFIELD, J.L. \& STEWART, B.A., eds., Soil biology. Effects on soil quality. Boca Raton, CRC Press, 1994. p.6183.

CROFT, M.; ROCHEFORT, L. \& BEAUCHAMP, C.J . Vaccuumextration of peatlands disturbs bacterial population and microbial biomass carbon. Appl. Soil Ecol., 18:1-12, 2001.

D'ANDRÉA, A.F.; SILVA, M.L.N.; CURI, N.; SIQUEIRA, J O. $\&$ CARNEIRO, M.A.C. Atributos biológicos indicadores da qualidade do solo em sistemas de manejo na região do cerrado no sul do Estado de Goiás. R. Bras. Ci. Solo, 26:913923, 2002.

FERREIRA, A.S.; CAMARGO, F.A.O. \& VIDOR, C. Utilização demicroondas na avaliação da biomassa microbiana do solo. R. Bras. Ci. Solo, 23:991-996, 1999.

FRIGHETTO, R.T.S. ??XVIII??. Análise da biomassa microbiana em carbono: método de fumigação-extração. In: FRIGHETTO, R.T.S. \& VALARINI, P.J., coords. Indicadores biológicos e bioquímicos da qualidade do solo. J aguariúna, EMBRAPA, 2000. p.157-166.

GHANI, A. \& WARDLE, D.A. Fate of ${ }^{14} \mathrm{C}$ from glucose and the herbicide metsulfuron-methyl in a plant-soil microcosm system. Soil Biol. Biochem., 33:777-785, 2001.

HARDEN, T.; J OERGENSEN, R.G.; MEYER, B. \& WOLTERS, $V$. Soil microbial biomass estimated by fumigationextraction and substrate-induced respiration in two pesticide-treated soils. Soil Biol. Biochem., 25:679-683, 1993.

HU, S.J .; van BRUGGEN, A.H.C. \& GRÜNWALD, N.J . Dynamics of bacterial populations in relation to carbon availability in a residue-amended soil. Appl. Soil Ecol., 13:21-30, 1999.

ISLAM, K.R. \& WEIL, R.R. Microwave irradiation of soil for routine measurement of microbial biomass carbon. Biol. Fertil. Soils, 27:408-416, 1998.

JENKINSON, D.S. \& POWLSON, D.S. The effects of biocidal treatments on metabolism in soil-I. Fumigation with chloroform. Soil Biol. Biochem., 8:167-177, 1976. 
LIN, Q. \& BROOKES, P.C. Comparison of substrate induced respiration, selective inhibition and biovolume of microbial biomass and its community structure in unamended, ryegrass-amended, fumigated and pesticide-treated soils. Soil Biol. Biochem., 31:1999-2114, 1999.

MOORMAN, T.B. Pesticide degradation by soil microorganisms: environmental, ecological, and management effects. In: HATFIELD, J.L. \& STEWART, B.A., eds. Soil biology. Effects on soil quality. Boca Raton, CRC Press, 1994. p.121169.

NIELSEN, M.N. \& WINDING, A. Microorganisms as indicators of soil health. Denmark, National Environmental Research Institute, 2002. 84p. (Technical Report, 388).

OLIVEIRA, J R.A.; MENDES, L.C. \& VIVALDI, L. Carbono da biomassa microbiana em solos de cerrado sob vegetação nativa e sob cultivo: avaliação dos métodos de fumigaçãoincubação e fumigação-extração. R. Bras. Ci. Solo, 25:863871, 2001.

PAGGA, U. Testing biodegradability with standardized methods. Chemosphere, 35:2953-2972, 1997.
PASCUAL, J .A.; GARCIA, C.; HERNADEZ, T.; MORENO, J .L. $\&$ ROS, M. Soil microbial activity as a biomaker of degradation and remediation processes. Soil Biol. Biochem., 32:1877-1883, 2000.

PAUL, E.A. \& CLARK, F.E. Soil microbiology and biochemistry. 2.ed. London, Academic Press, 1996. p.109-127.

SCHINNER, F.; ÖHLINGER, R.; KANDELER,E.\& MARGESIN, R., eds. Indirect estimation of microbial biomass. In: Methods in soil biology. Heidel berg, Springer-Verlag, 1996. p.47-75.

SPARLING, G.P.; FELTHAM, C.W.; REYNOLDS, J .; WEST, A.W. \& SINGLETON, P. Estimation of soil microbial C by fumigation-extraction method: use on soils of high organic matter content, and reassessment of the $\mathrm{kec}_{\mathrm{ec}}$ factor. Soil Biol. Biochem., 22:301-307, 1990.

SUETT, D.L.; FOURNIER, J.C.; MOURKIDOU, E.P.; PUSSEMIER, L. \& SMELT, J. Accelerated degradation: the European dimension. Soil Biol. Biochem., 28:1741-1748, 1996.

VANCE, E.D.; BROOKES, P.C. \& JENKINSON, D.S. An extraction method for measuring soil microbial biomass. Soil Biol. Biochem., 19:703-707, 1987. 